Proceedings of the 13th International Symposium UFPS, Vilnius, Lithuania 2007

\title{
Monte Carlo Treatment of Non-Equilibrium Processes in $n$-Type InSb Crystals
}

\author{
S. Ašmontas And R. RAGuOtis* \\ Semiconductor Physics Institute, Goštauto 11, LT-01108 Vilnius, Lithuania \\ Numerical calculation by Monte Carlo method of the dynamic behaviour \\ of electron ensemble in $n$-type InSb crystals after step-like application of elec- \\ tric field is presented. The results show essential influence of electron density \\ on the energy relaxation time. The effect of electron energy cooling below \\ equilibrium temperature in compensated $n$-InSb is obtained numerically for \\ the first time, which is in agreement with experimental results.
}

PACS numbers: 52.65.Pp, 72.10.-d, 72.10.Fk, 72.20.Dp

\section{Introduction}

The energy relaxation time dependence on electron density in $n$-type InSb at lattice temperature $T=78 \mathrm{~K}$ has been measured in [1]. An interesting effect of electron gas cooling below the equilibrium value in highly compensated $n$-InSb was obtained experimentally.

In the presented work the effect is analysed by Monte Carlo (MC) methods. For this purpose we used a modified combined scattering rate (CSR) MC method [2] and ensemble (EMC) MC method [3]. CSR method is based on a concept of ensemble events and avoids the short-time-step procedure inherent to conventional EMC methods. It is especially convenient when electron-electron (e-e) scattering is included.

\section{Results and discussion}

$n$-InSb parameters used in the calculation were similar to those used in $[4,5]$ : effective mass ratio 0.014 , density $5.79 \mathrm{~g} / \mathrm{cm}^{3}$, sound velocity $3.7 \times 10^{5} \mathrm{~m} / \mathrm{s}$, acoustic deformation potential $30 \mathrm{eV}$, optical phonon energy $25 \mathrm{meV}$, static and high-frequency dielectric constants were correspondingly 17.9 and 15.7. Inasmuch as electric field is small we have neglected conduction band non-parabolicity.

At first we shall discuss the results of simulation of electron ensemble time response to a pulse of comparatively small electric field amplitude. Here for better understanding of role played by e-e scattering in transport phenomena we have calculated the response of a pure semiconductor, i.e. when the impurity scattering is negligible. The obtained results are shown in Fig. 1. It can be noted that the

${ }^{*}$ corresponding author; e-mail: romasr@pfi.lt 

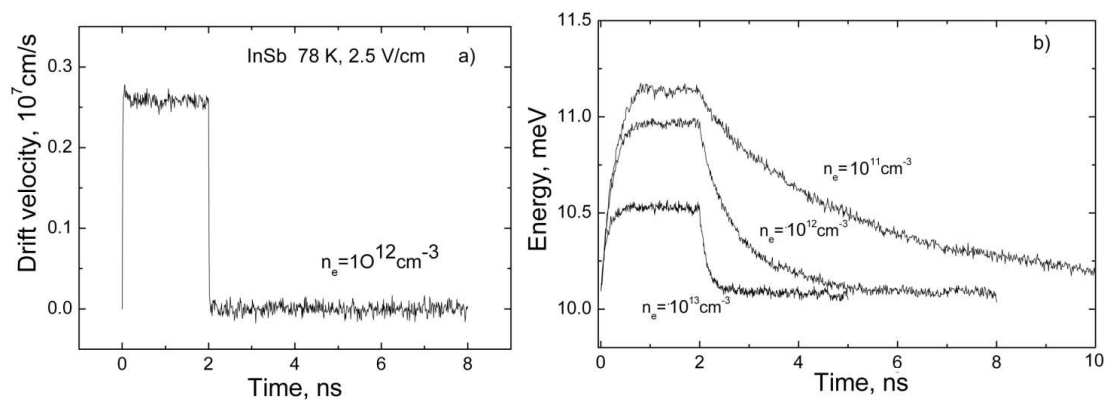

Fig. 1. Electron response time in $n$-type $\operatorname{InSb}$ at $T=78 \mathrm{~K}$. The probe electric field pulse $E=2.5 \mathrm{~V} / \mathrm{cm}$. The pulse duration is $2000 \mathrm{ps}$, then the electric field is switched off. (a) Drift velocity, (b) energy.

drift velocity response weakly depends on electron density $n_{\mathrm{e}}$. Therefore, only a single curve obtained for $n_{\mathrm{e}}=10^{12} \mathrm{~cm}^{-3}$ is shown. In the scale the drift velocity achieves maximum value practically instantaneously and remains constant during pulse action. The behaviour of the mean energy substantially differs, Fig. 1b. Here an essential influence of electron density on the response is seen. The maximum value of the energy strongly diminishes with $n_{\mathrm{e}}$. At used field, $E=2.5 \mathrm{~V} / \mathrm{cm}$, the electrons mean energy is smaller than optical phonon energy. When the electron density is small, then after the field is switched off the electrons mainly relax due to acoustic phonons emission, which is quasi-elastic in energy. When $n_{\mathrm{e}}$ increases, e e scattering favours for electrons to lose their energy due to mutual mixing between passive and active areas divided by optical phonon energy.

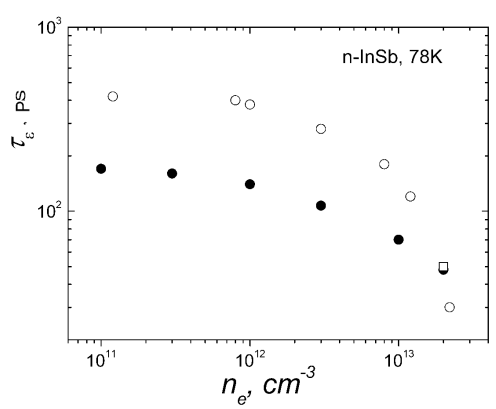

Fig. 2. Energy relaxation time versus electron density. Experiment: open circles [1], open square [6]. Closed circles - MC simulation.

The above results can serve for determination of the phenomenological electron energy relaxation time $\tau_{\varepsilon}$, which can be obtained from energy balance rate equation: $\tau_{\varepsilon}=\left(\varepsilon-\varepsilon_{0}\right) / e v_{\mathrm{d}} E$, where $\varepsilon$ and $\varepsilon_{0}$ are heated and equilibrium electron mean energies, $e$ is electron charge, $v_{\mathrm{d}}$ is steady-state drift velocity at field $E$. The corresponding results together with experimental ones are plotted in Fig. 2. It is 
seen that the results obtained by MC method are about twice smaller, however the trends are similar. But it is well known that $n$-InSb is strongly a non-linear semiconductor, therefore, small amplitudes of the field, as in case our simulation, do not belong to ohmic region. If we will take lower field values we should obtain better agreement but MC method is imprecise at low fields. Nevertheless, these results together with those plotted in Fig. 1 give some understanding of the role of e-e collisions in the transport phenomena.

An interesting effect of electron gas cooling in highly compensated $n$-InSb was obtained experimentally in [1]. This effect appears when electron energy scattering is dominated by optical phonon emission, while momentum loss is ruled by impurity scattering. Electrons accelerating by electric field reach optical phonon energy $\hbar \omega_{0}$, then they instantaneously emit optical phonon and settle down in the state with $\varepsilon \approx 0$, and due to intensive impurity scattering at low energies the electrons localise at this condition for a quite long. This leads to the situation where the total electron energy can be somewhat smaller than the equilibrium one. This effect has been predicted analytically in Ref. [7].

We performed corresponding MC calculation. For the simplicity the impurity scattering modelling was performed within the Brooks-Herring relaxation time approximation [8]. As in [1] we took impurity density equal to $2 \times 10^{15} \mathrm{~cm}^{-3}$ and free electron concentration $10^{11} \mathrm{~cm}^{-3}$. Due to the small electron density e-e scattering has been neglected and calculations were performed by conventional EMC method [3].
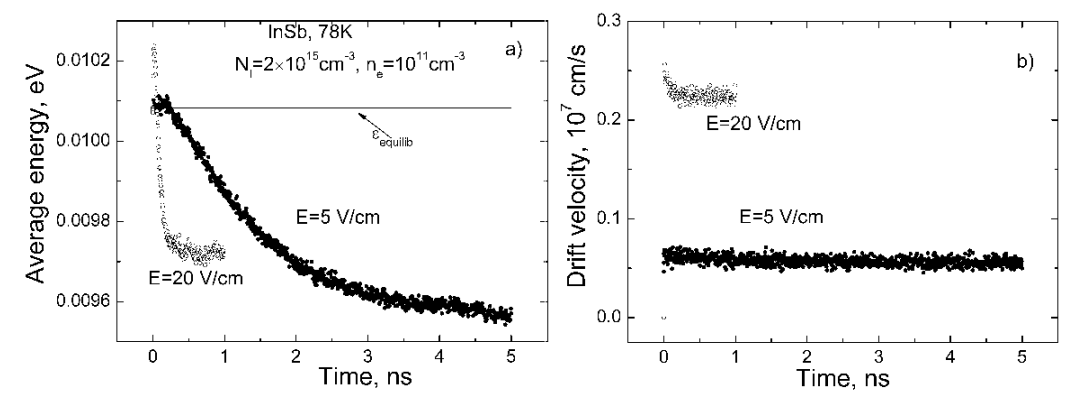

Fig. 3. Time response of average energy (a) and drift velocity (b) in $n$-type InSb at $T=78 \mathrm{~K}$ after step-like switched on electric field.

The results of calculation of electron ensemble response are shown in Fig. 3 for two values of electric field $\approx 5$ and $20 \mathrm{~V} / \mathrm{cm}$. Here we see that the mean electron energy after overshoot decreases to the values lower that the equilibrium one. At the same time drift velocity response has a conventional behaviour.

Electron cooling vanishes when electron density is increased [1, 7]. To test this we made corresponding MC simulation. According to experiment we took a compensated case: $n_{\mathrm{e}}=10^{15} \mathrm{~cm}^{-3}$ and $N_{\mathrm{I}}=2 \times 10^{15} \mathrm{~cm}^{-3}$. The simulation 


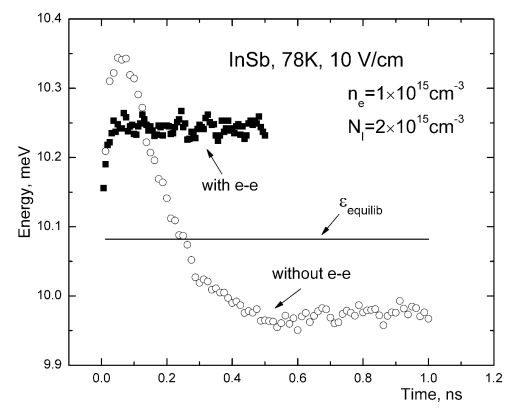

Fig. 4. Time response of average energy in $n$-type $\operatorname{InSb}$ at $T=78 \mathrm{~K}$ after step-like switched on electric field. $E=10 \mathrm{~V} \mathrm{~cm}$.

was made for the situation when both e-e and impurity scattering were included and when only impurity scattering was left. We must note that in calculating the last case we took for the screening constant the same value as in the first case. This can be justified by the reasoning that the inverse screening constant for $n_{\mathrm{e}}=10^{15} \mathrm{~cm}^{-3}$ is about $10^{9} \mathrm{~cm}^{-1}$, when the reverse mean distant between impurities for given $N_{\mathrm{I}}$ has the same order.

From Fig. 4 it is seen that e-e scattering removes electron cooling. This result coincides with the results obtained experimentally in [1] and predicted theoretically in [7].

\section{Conclusions}

We have investigated the response of electron ensemble to a pulse of electric field in $n$-type InSb crystals by MC method. The obtained results show significant influence of electron density on the electron energy relaxation time. The results obtained by numerical MC calculations show the possibility of electron gas cooling in a compensated semiconductor.

\section{References}

[1] S. Ašmontas, Electrogradient Phenomena in Semiconductors, Vilnius, Mokslas 1984 (in Russian).

[2] A. Matulionis, R. Raguotis, R. Katilius, Phys. Rev. B 56, 2052 (1997).

[3] C. Jacoboni, P. Lugli, The Monte Carlo Method for Semiconductor Device Simulation, Springer, New York 1989.

[4] A. Krotkus, Z. Dobrovolskis, Electron Conduction of Narrow-Gap Semiconductors, Vilnius, Mokslas 1988 (in Russian).

[5] M. Akarsu, Ö. Özbaş, Turk. J. Phys. 26, 283 (2002).

[6] V. Dienys, Z. Martūnas, G. Tvardauskas, Fiz. Techn. Poluprovodn. 16, 1856 (1982).

[7] Z.S. Gribnikov, V.A. Kochelap, Zh. Eksp. Teor. Fiz. 58, 1046 (1970).

[8] B.K. Ridley, Quantum Process in Semiconductors, Clarendon Press, Oxford 1982. 